\title{
REGULAR CURVES ON RIEMANNIAN MANIFOLDS $\left({ }^{1}\right)$
}

\author{
BY \\ STEPHEN SMALE
}

Introduction. A regular curve on a Riemannian manifold is a curve with a continuously turning nontrivial tangent vector. $\left({ }^{2}\right)$ A regular homotopy is a homotopy which at every stage is a regular curve, keeps end points and directions fixed and such that the tangent vector moves continuously with the homotopy. A regular curve is closed if its initial point and tangent coincides with its end point and tangent. In 1937 Hassler Whitney [17] classified the closed regular curves in the plane according to equivalence under regular homotopy. The main goal of this work is to extend this result to regular curves on Riemannian manifolds.

THEOREM A. Let $x_{0}$ be a point of the unit tangent bundle $T$ of a Riemannian manifold $M$. Then there is a 1-1 correspondence between the set $\pi_{0}$ of classes (under regular homotopy) of regular curves on $M$ which start and end at the point and direction determined by $x_{0}$ and $\pi_{1}\left(T, x_{0}\right)$.

This correspondence may be described as follows. If $\bar{f} \in \pi_{0}$ let $f$ be a representative of $\bar{f}$ and let $\bar{\phi} f$ at $t$ be the vector of $T$ whose base point is $f(t)$ and whose direction is defined by $f^{\prime}(t)$, the derivative of $f$ at $t$. Then $\bar{\phi} f$ is a curve on $T$ which represents an element of $\pi_{1}\left(T, x_{0}\right)$. The correspondence of Theorem $\mathrm{A}$ is that induced by $\bar{\phi}$.

If $f$ is a closed regular curve in the plane then its rotation number $\gamma(f)$ is the total angle which $f^{\prime}(t)$ turns as $t$ traverses $I$. The Whitney-Graustein Theorem says that two closed regular curves on the plane are regularly homotopic if and only if they have the same rotation number. Using the fact that the unit tangent bundle of the plane is $E^{2} \times S^{1}$, this theorem follows from Theorem A.

Let $x_{0}$ be a point of the unit tangent bundle $T$ of a Riemannian manifold $M$. The space of all regular curves on $M$ starting at the point and direction determined by $x_{0}$ is denoted by $E$. A map $\pi$ from $E$ onto $T$ is defined by sending a curve into the tangent of its endpoint at its endpoint. The following can be considered as the fundamental theorem of this work.

Theorem B. The triple $(E, \pi, T)$ has the covering homotopy property for polyhedra.

Presented to the Society, August 24, 1956; received by the editors September 29, 1956.

(1) The material in this paper is essentially a dissertation submitted in partial fulfillment of the requirements for the degree of Doctor of Philosophy in the University of Michigan, 1956.

(2) These definitions will be made precise in the body of the work. Also, theorems stated will be proved later. 
Let $\Gamma$ be the fiber over $x_{0}$ of $(E, \pi, T)$ and let $\Omega$ be the ordinary loop space of $T$ at $x_{0}$.

Theorem C. The map $\Phi$ is a weak homotopy equivalence between $\Gamma$ and $\Omega$.

Theorem B is used to obtain Theorem C and, in turn, Theorem A follows from Theorem $\mathrm{C}$.

If $f$ is a regular curve on $M, \bar{\phi} f$ is a curve, as we shall say, a lifted curve, on $T=T(M)$. Clearly, not all curves on $T$ are lifted curves. In particular, every lifted curve must be an integral curve of a certain 1-form $\omega_{0}$ on $T$. If the integral curves of $\omega_{0}$ were exactly the lifted curves, Theorems A, B, and $C$ could be proved by proving theorems on integral curves. Unfortunately, however, $\omega_{0}$ admits as integral curves some non-lifted curves. Nevertheless, these considerations raise questions concerning the loop space of integral curves of $\omega_{0}$ on $T$.

Theorem D. Let $\omega$ be a 1-form of Class $A$ on a three dimensional manifold $M$ such that $\omega \wedge d \omega \neq 0$ on $M$ and let $x_{0} \in M$. Denote by $\Omega_{\omega}$ the loop space at $x_{0}$ of piecewise regular curves on $M$ which are integral curves of $\omega$ and by $\Omega$ the ordinary loop space of $M$ at $x_{0}$. Then the inclusion $i: \Omega_{\omega} \rightarrow \Omega$ is a weak homotopy equivalence.

I would like to express my appreciation to my adviser, Professor Raoul Bott, for the encouragement and advice which he gave throughout the preparation of this thesis. I would also like to thank Professor Hans Samelson for reading the manuscript and for suggesting several corrections.

1. Fiber spaces. A triple $(E, p, B)$ will consist of two arcwise connected spaces $E, B$ and a map $p$ from $E$ in to $B$. A triple will be said to have the CHP if it has the covering homotopy property for polyhedrons [11].

If $g$ is a map from a space $X$ into a space $Y$, then the restriction of $g$ to a subset $A$ of $X$ will be denoted by $g_{\mid A}$ or sometimes just $g$. $P$ will always denote a polyhedron. The set $\{t$ real $\mid a \leqq t \leqq b\}$ is denoted by $[a, b]$. A cube $I^{k}$ is the Cartesian product of $k$ copies of $I$, the closed unit interval.

The following proposition is well-known. It is a special case of a theorem proved in $[6$, p. 136].

Proposition 1.1. Let $(E, p, B)$ be a triple which has the covering homotopy property for cubes. Then it also has the CHP.

Lemma 1.2. Let a triple $(E, p, B)$ have the CHP. Let $\sigma$ be a simplex of some dimension $n$ and let $g: \sigma \times I \rightarrow B$ be given. Suppose $\dot{\sigma}$ is the (pointset) boundary of $\sigma, A=\dot{\sigma} \times I \cup \sigma \times 0 \subset \sigma \times I$ and $f: A \rightarrow E$ covers $g_{\mid A}$. Then there exists an extension $F$ of $f$ to all of $\sigma \times I$ covering $g$.

The proof is immediate. There is a homeomorphism from $\sigma \times I$ onto $I^{n} \times I$ which sends $A$ homeomorphically onto $I^{n} \times 0$. Then the application of the CHP yields the desired map $F$. 
The statement of the following theorem is quite similar to what Hurewicz calls the Uniformization Theorem in [5]. It will be found useful in proving the $\mathrm{CHP}$ for a triple whose base space is a manifold.

Proposition 1.3. Suppose a triple $(E, p, B)$ has the CHP locally; that is, for each point $x \in B$, there exists a neighborhood $V$ of $x$ such that $\left(p^{-1}(V), p, V\right)$ has the CHP. Then $(E, p, B)$ has the CHP.

Proof. Let $H: P \times I \rightarrow B$ be a given homotopy and $h: P \times 0 \rightarrow E$ a covering of $H_{\mid P \times 0}$. We will define a covering homotopy $\bar{H}: P \times I \rightarrow E$. For each $y \in H(P \times I)$ let $V_{y}$ be a neighborhood of $y$ so that $\left(p^{-1}\left(V_{y}\right), p, V_{y}\right)$ has the CHP. Assume $P \times I$ has been given some definite metric. Denote by $\delta$ the Lebesgue number of the covering $\left\{U_{y}=H^{-1}\left(V_{y}\right) \mid y \in H(P \times I)\right\}$ of $P \times I$. Put $I_{0}=[0, \delta / 3]$. It is sufficient to define $\bar{H}$ on $P \times I_{0}$ for then iteration will yield a full covering homotopy.

Take a simplicial complex $K$ such that $|K|=P$. Let $\operatorname{Sd}(K)$ be a subdivision of $K$ such that the diameter of any simplex of $\operatorname{Sd}(K)$ is less than $\delta / 3$, and let $\operatorname{Sd}(K)^{r}$ be the $r$-skeleton of $\operatorname{Sd}(K)$. If $v$ is a vertex of $\operatorname{Sd}(K)$ the choice of $I_{0}$ yields that $H\left(v \times I_{0}\right)$ is contained in some neighborhood $V$ where $\left(p^{-1}(V), p, V\right)$ has the CHP. This fact immediately gives a definition of $\bar{H}$ on $\left|\operatorname{Sd}(K)^{0}\right| \times I_{0}$. Proceeding by induction suppose $\bar{H}$ has been defined on $\operatorname{Sd}(K)^{r-1} \mid \times I_{0}$, and $\sigma^{r}$ is an $r$-simplex of $\operatorname{Sd}(K)$. From the choices of $\operatorname{Sd}(K)$ and $I_{0}$ it follows that $\sigma^{r} \times I_{0}$ has diameter less than $\delta$. Then $H\left(\sigma^{r} \times I_{0}\right)$ is contained in some $V$ such that $\left(p^{-1}(V), p, V\right)$ has the CHP. Already $\bar{H}$ has been defined on $\dot{\sigma}^{r} \times I_{0} \cup \sigma^{r} \times 0$. Then application of Lemma 1.2 yields $\bar{H}$ on $\sigma^{r} \times I_{0}$. In this manner $\bar{H}$ is defined on all of $\left|\operatorname{Sd}(K)^{r}\right| \times I_{0}$ and then by induction on all of $|\operatorname{Sd}(K)| \times I_{0}$. This proves 1.3.

2. Regular curves. By a manifold we shall mean a connected Riemannian manifold of class 3 and dimension greater than 1 . There are no assumptions such as completeness or compactness. If $M$ is a manifold, $T_{0}(M)$ or often just $T_{0}$ will be the space (or bundle) of all tangent vectors of $M$. By $T(M)$ or $T$ is meant the sub-bundle of $T_{0}$ which consists of the unit tangent vectors of $M$.

As usual, a curve on a space is a map of $I$ into the space. Let $M$ be a manifold and let $f$ be a curve on $M$ whose derivative $[1, \mathrm{p} .46]$ exists at the value $t_{0} \in I$. This derivative is an element $v\left(t_{0}\right)$ of $M_{f\left(t_{0}\right)}$, the tangent vector space of $M$ at $f\left(t_{0}\right)$. By convention in this work the derivative $f^{\prime}\left(t_{0}\right)$ of $f$ at $t_{0}$ will be the pair $\left(f\left(t_{0}\right), v\left(t_{0}\right)\right)$. Thus, $f^{\prime}\left(t_{0}\right)$ will be an element of $T_{0}(M)$. By the magnitude of $f^{\prime}\left(t_{0}\right)$ or $\left|f^{\prime}\left(t_{0}\right)\right|$ we mean the magnitude of $v\left(t_{0}\right)$.

A parametrized regular curve on a manifold $M$ is a curve $f$ on $M$ such that $f^{\prime}(t)$ exists, is continuous and has positive magnitude for each $t \in I$. Two parametrized regular curves $f$ and $g$ will be called equivalent if there exists a homeomorphism $h$ of $I$ onto itself such that for all $t \in I, h^{\prime}(t)$ exists, is continuous and positive, and $f(t)=g(h(t))$. It is an easy matter to check that this 
is a true equivalence. A regular curve is an equivalence class of parametrized regular curves.

The arc-length of a regular curve $g$, defined in the usual way, exists, and is independent of its representative. It will be denoted by $L(\mathrm{~g})$. Implicit use of the following proposition will be made throughout this work.

PROPOSITION 2.1. If $g$ is a regular curve on a manifold then there exists a unique representative of $g$ still denoted by $g$ such that $\left|g^{\prime}(t)\right|=L(g)$ for all $t \in I$. $L(g)$ is the only possible constant value here.

The proof for the plane is in [17]. The same proof holds for the general case of a manifold. The representative of a regular curve given by 2.1 will be called distinguished. A distinguished representative is just a parametrization proportional to arc-length. Unless we note otherwise, a regular curve will be identified with its distinguished representative.

Let $M$ be a manifold and $x_{0}$ a fixed point of $T(M)$. We denote by $E(M)$ or sometimes simply $E$, the space of regular curves on $M$ whose normalized initial tangents are $x_{0}$; in other words,

$$
E(M)=\left\{g \text { is a regular curve on } M \mid \frac{g^{\prime}(0)}{\left|g^{\prime}(0)\right|}=x_{0}\right\} .
$$

Let $\bar{d}: T_{0} \times T_{0} \rightarrow R^{+}$be any metric on $T_{0}\left(R^{+}\right.$is the space of non-negative real numbers). Then for $f$ and $g \in E$, let

$$
d(f, g)=\max \left\{\bar{d}\left[f^{\prime}(t), g^{\prime}(t)\right] \mid t \in I\right\} .
$$

From the fact that $\bar{d}$ is a metric, it follows easily that $d$ is a metric on $E$. We will suppose $E$ to have the topology induced by $d$.

Let $f_{i}$ be a sequence of points of $E$ converging to a point $f$ of $E$. Then for each $t \in I, f_{i}^{\prime}(t)$ converges to $f^{\prime}(t)$. If a sequence $x_{n}$ of points of $T_{0}$ converges to $x_{0}$ then from the topology of $T_{0}$ it follows that the base points of $x_{n}$ in $M$ converge to the base point of $x_{0}$. Thus $f_{i}(t)$ converges to $f(t)$ for each $t \in I$.

The map $\pi: E \rightarrow T$ of Theorem B may be defined by $\pi(f)=f^{\prime}(1) /\left|f^{\prime}(1)\right|$. To speak of $(E, \pi, T)$ as a triple, $E$ must be arcwise connected. This is proved later (Lemma 6.2).

3. The reduction of the proof of Theorem B to 3.1 and 3.2. The proof of Theorem B depends essentially on Propositions 3.1 and 3.2.

Proposition 3.1. Let $M$ be a manifold and $p: E(M) \rightarrow M$ be the map $p(g)$ $=g(1)$. Then $(E, \bar{p}, M)$ has the CHP.

Suppose $M$ is a manifold. Let $\pi_{1}: T_{0}(M) \rightarrow M$ be the map which sends a tangent vector into its base point. A homotopy $f_{v}: P \rightarrow T_{0}(M)$ will be called vertical if for all $p \in P$ and $v \in I, \pi_{1} f_{v}(p)=\pi_{1} f_{0}(p)$. A homotopy $f_{v}: P \rightarrow X$ ( $X$ any space) is said to be stationary on a subpolyhedron $A$ of $P$ if $f_{v}(p)$ $=f_{0}(p)$ for all $p \in A$ and $v \in I$. 
Proposition 3.2. Let $M$ be a manifold and $f_{v}: P \rightarrow T(M)$ be a given vertical homotopy with $\bar{f}: P \rightarrow E(M)$ covering $f_{0}$. Then there exists a covering homotopy $\bar{f}_{v}: P \rightarrow E$. Furthermore if $f_{v}$ is stationary on a subpolyhedron $A$ of $P$ then $\bar{f}_{v}$ will also be.

Propositions 3.1 and 3.2 will be proved in the following sections. Now we will show how Theorem B follows from 3.1 and 3.2.

Lemma 3.3. Let $P$ be a polyhedron and $A$ be a subpolyhedron which is a strong deformation retract [2] of $P$. Let $g$ and $h$ be maps of $P$ into a space $X$ which agree on $A$. Then there exists a homotopy $H: P \times I \rightarrow X$ between $g$ and $h$ which is stationary on $A$.

Proof. Since $A$ is a strong deformation retract of $P$ there is a homotopy $K: P \times I \rightarrow P$ such that $K(p, 0)=p, K(p, 1) \in A$, and if $p \in A, K(p, t)=p$. The desired homotopy $H: P \times I \rightarrow X$ may be defined as follows:

$$
\begin{array}{ll}
H(p, t)=g K(p, 2 t) & 0 \leqq t \leqq 1 / 2, \\
H(p, t)=h K(p, 2-2 t) & 1 / 2 \leqq t \leqq 1 .
\end{array}
$$

The $k$-sphere is denoted by $S^{k}$.

Lemma 3.4. Let $P$ and $A$ be as above with $P$ contractible, and $M$ be an $n$ dimensional manifold. Let $F: P \rightarrow M$ be given and $g: P \rightarrow T=T(M), h: P \rightarrow T$ be two covering maps of $F$ which agree on $A$. Then there exists a homotopy $h_{v}: P \rightarrow T$ between $g$ and $h$ such that $h_{v}$ is stationary on $A$ and for each $v \in I, h_{v}$ covers $F$.

Proof. Let $E^{\prime}$ be the induced bundle $F^{-1}(T)[13$, p. 47$]$.

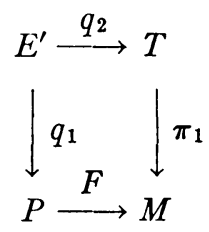

By definition:

$$
\begin{array}{ll}
E^{\prime}=\left\{(p, t) \in P \times T \mid F(p)=\pi_{1}(t)\right\} . \\
q_{2}(p, t)=t, & q_{1}(p, t)=p .
\end{array}
$$

Since $P$ is contractible, $E^{\prime}$ is a product $P \times S^{n-1}\left[13\right.$, p. 53] with $q_{1}$ being the projection of $E^{\prime}$ onto $P$. Let $\pi^{\prime}: E^{\prime} \rightarrow S^{n-1}$ be the other projection.

Define $\bar{g}: P \rightarrow E^{\prime} \subset P \times T$ by $\bar{g}(p)=(p, g(p))$ and let $\bar{g}^{*}: P \rightarrow S^{n-1}$ be the composition $\pi^{\prime} \bar{g}$. Similarly, define $\bar{h}$ and $\bar{h}^{*}$ from $h$.

Apply the previous lemma to obtain a homotopy $\bar{h}_{0}^{*}: P \rightarrow S^{n-1}$ between $\bar{g}^{*}$ and $\bar{h}^{*}$ which is stationary on $A$. Define $\bar{h}_{v}: P \rightarrow E^{\prime}=P \times S^{n-1}$ by $\bar{h}_{v}(p)$ $=\left(p, \bar{h}_{v}^{*}(p)\right)$ and $h_{v}: P \rightarrow T$ by $h_{v}=q_{2} \bar{h}_{v}$. It can be quickly checked that $h_{v}$ 
satisfies the lemma. q.e.d.

To prove Theorem B it is sufficient by Proposition 1.1 to show that $(E, \pi, T)$ has the covering homotopy property for cubes. Suppose, then, we are given a homotopy $f_{v}: P \rightarrow T$ and a map $\bar{f}: P \rightarrow E$ covering $f_{0}$ where $P$ is a cube. We will construct a covering homotopy $\bar{f}_{v}: P \rightarrow E$.

Application of Proposition 3.1 yields a covering map $\bar{h}: P \times I \rightarrow E$ of $\pi_{1} f_{v}$ such that $\bar{h}(p, 0)=\bar{f}(p)$. Lemma 3.4 then yields a homotopy $H_{u}: P \times I \rightarrow T$ such that $(1) H_{0}(p, v)=\pi \bar{h}(p, v)$, (2) $H_{1}(p, v)=f_{v}(p)$, (3) $H_{u}(p, v)$ covers $\pi_{1} f_{v}(p)$ for each $u \in I$, and (4) $H_{u}$ is stationary on $P \times 0$. By (3) $H_{u}$ is a vertical homotopy so Proposition 3.2 applies to yield a homotopy $\bar{H}_{u}: P \times I \rightarrow E$ of $\bar{h}$ which covers $H_{u}$.

We assert that $\bar{H}_{1}(p, v)$ can be taken as the desired covering homotopy $\bar{f}_{v}(p)$. From $H_{1}(p, v)=f_{v}(p)$ it follows that $\bar{H}_{1}(v, p)$ covers $f_{v}(p)$. Since $H_{u}$ is stationary on $P \times 0, \bar{H}_{u}$ is also. Then $\bar{H}_{1}(p, 0)=\vec{H}_{0}(p, 0)=\bar{h}(p, 0)=\bar{f}(p)$ or $\bar{H}_{1}(p, v)$ is a homotopy of $\bar{f}(p)$. This shows that Theorem $\mathrm{B}$ follows from Propositions 3.1 and 3.2.

4. Proof of Proposition 3.1. We will need two lemmas. By $v \perp w$ it is meant that the vectors $v$ and $w$ are perpendicular.

Lemma 4.1. Let $n>1$ and $S^{n-1}$ be the unit vectors of Euclidean $n$-space $E^{n}$ considered as a vector space. Suppose $P$ is a cube and a map $w: P \rightarrow S^{n-1}$ is given. Then there exists a map $u: P \rightarrow S^{n-1}$ such that for all $p \in P, u(p) \perp w(p)$.

This lemma is not true for a general polyhedron. In particular, if $P=S^{n-1}$ and $w$ is the identity, the existence of such a map $u$ implies the existence of a unit vector field on $S^{n-1}$. It is well known that this is impossible for odd $n$.

Proof of 4.1. Let $V_{n, 2}$ be the Stiefel manifold [13, p. 33] of ordered orthogonal unit 2 -frames in $E^{n}$. With a projection $p_{1}$ sending a 2 -frame into its first vector, $V_{n, 2}$ becomes an $(n-2)$-sphere bundle over $S^{n-1}$. Let $E^{\prime}$ be the induced bundle $w^{-1}\left(V_{n, 2}\right)$.

$$
\stackrel{f}{E^{\prime}} \stackrel{f}{\rightarrow} V_{n, 2}
$$

Since $P$ is contractible $E^{\prime}$ is a product. I.et $s: P \rightarrow E^{\prime}$ be any cross-section, and let $p_{2}: V_{n, 2} \rightarrow S^{n-1}$ send a 2 -frame into its second vector. Then the composition $u=p_{2} f s$ has the desired property. q.e.d.

I.емм 4.2. Given $y_{0}, 0<y_{0} \leqq 1 / 2$, there exists a real continuous differentiable function $\beta(y)$ defined on I such that (1) $\beta(0)=\beta(1)=0$, (2) $\beta^{\prime}(0)=0$, and (3) for $y_{0} \leqq y \leqq 1, \beta^{\prime}(y) \geqq 4$.

Proof. Consider the function: 


$$
\begin{array}{lrl}
r(y) & =0 & 0 \leqq y \leqq y_{0} / 2, \\
r(y) & =-\frac{8}{y_{0}}\left(1-y_{0}\right) y+r\left(1-y_{0}\right) & y_{0} / 2 \leqq y \leqq y_{0}, \\
r(y) & =4 y-4 & y_{0} \leqq y \leqq 1 .
\end{array}
$$

Note that $r(y)$ could be taken as $\beta(y)$ except for the fact that it has corners at $y=y_{0} / 2$ and $y=y_{0}$. By "rounding off the corners" of $r(y)$ the desired function can be obtained.

In order to prove 3.1 it is sufficient by Proposition 1.3 to show that $\left(\bar{p}^{-1}\left(U_{0}\right), \overline{ }, U_{0}\right)$ has the CHP where $U_{0}$ is a coordinate neighborhood of $M$. Since $U_{0}$ is homeomorphic to $E^{n}$ we can identify the two spaces under this homeomorphism. Thus $U_{0}=E^{n} \subset M . T_{0}\left(U_{0}\right)$ is a product space $E^{n} \times E^{n}$ where the first factor comes from the base point and the second from the direction and magnitude of a vector. We identify each of the two factors of $T_{0}\left(U_{0}\right)$ with a single $E^{n}$ whose elements we consider as vectors. If $S^{n-1}$ is the space of unit vectors of $E^{n}, T\left(U_{0}\right)=E^{n} \times S^{n-1}$. The magnitude of a vector $v$ of $E^{n}$ is written $|v|$.

For convenience the following new convention is used in this section and the next. The derivative of a regular curve at a point of $U_{0}$ will not carry the base point. That is, it is now the projection of the old derivative onto the second factor of $T_{0}\left(U_{0}\right)$. This is possible since most of the analysis in these sections is concerned with $T_{0}\left(U_{0}\right)$ and $U_{0}$.

By Proposition 1.1 it is enough to show that $\left(\bar{p}^{-1}\left(U_{0}\right), \overline{ }, U_{0}\right)$ has the covering homotopy property for cubes. Let $h_{v}: P \rightarrow U_{0}$ be a given homotopy with $\vec{h}: P \rightarrow \bar{p}^{-1}\left(U_{0}\right)$ covering $h_{0}$ where $P$ is a cube. We will construct a covering homotopy $\bar{h}_{v}: P \rightarrow \bar{p}^{-1}\left(U_{0}\right)$.

Choose $J$ with $0 \leqq J<1$ such that for all $p \in P$ and $t \in[J, 1], \vec{h}(p)(t) \in U_{0}$. Then choose $J_{0}$ with $J \leqq J_{0}<1$ so that for all $p \in P$ and $t \in\left[J_{0}, 1\right]$,

$$
\left|\bar{h}^{\prime}(p)(t)-\bar{h}^{\prime}(p)(1)\right|<\frac{\left|\bar{h}^{\prime}(p)(1)\right|}{10} .
$$

The following choices are motivated by the need to insure the regularity of the covering homotopy curves we are constructing. Let

$$
K=\max \left\{\left|h_{v}(p)-h_{0}(p)\right| \mid v \in I, p \in P\right\} .
$$

If $K=0, y_{0}=1 / 2$. Otherwise let

$$
y_{0}=\min \left\{\frac{1}{2}, \frac{\left|\bar{h}^{\prime}(p)(1)\right|\left(1-J_{0}\right)}{6 K} \mid p \in P\right\} .
$$

The compactness of $P$ yields that $y_{0}>0$.

Taking $y_{0}$ as above, let $\beta(y)$ be the function given by Lemma 4.2.

By taking $w(p)=\bar{h}^{\prime}(p)(1) /\left|\bar{h}^{\prime}(p)(1)\right|$, Lemma 4.1 yields a map $u: P \rightarrow S^{n-1}$ 
such that $u(p) \perp \bar{h}^{\prime}(p)(1)$.

We define the desired covering homotopy $\vec{h}_{v}: P \rightarrow \bar{p}^{-1}\left(U_{0}\right)$ as follows. For $0 \leqq t \leqq J_{0}$ set $\bar{h}_{v}(p)(t)=\bar{h}(p)(t)$. For $J_{0} \leqq t \leqq 1$ let $s=s(t)=\left(t-J_{0}\right) /\left(1-J_{0}\right)$; then set

$$
\bar{h}_{v}(p)(t)=\bar{h}(p)(t)+s^{2}\left[h_{v}(p)-h_{0}(p)\right]+\beta(s)\left|h_{v}(p)-h_{0}(p)\right| u(p) .
$$

Here $\bar{h}(p)$ is to be taken distinguished (see $\S 2$ ), but $\bar{h}_{v}(p)$, in general will not be. Note that for $t \geqq J_{0}$ all the terms used to define $\bar{h}_{v}(p)$ lie in $U_{0}$ and hence the additions make sense.

The following properties of $\bar{h}_{v}$ can be readily checked:

(1) $\bar{h}_{v}(p)(t)$ is continuous in $v, p$, and $t$.

(2) $\bar{h}_{v}(p)(1)=h_{v}(p)$.

(3) $\bar{h}_{0}(p)=\bar{h}(p)$.

The derivative of $\bar{h}_{v}(p)(t)$ for $t \geqq J_{0}$ can be computed to be:

$\bar{h}_{v}^{\prime}(p)(t)=\bar{h}^{\prime}(p)(t)+s^{\prime} 2 s\left[h_{v}(p)-h_{0}(p)\right]+s^{\prime} \beta^{\prime}(s)\left|h_{v}(p)-h_{0}(p)\right| u(p)$.

Then it can be seen:

(4) $\bar{h}_{v}(p)$ is differentiable.

(5) $\bar{h}_{v}^{\prime}(p)(0) /\left|\bar{h}_{v}^{\prime}(p)(0)\right|=x_{0}$. The derivative meant here is in the sense of $\S 2$.

The following requires proof:

(6) $\bar{h}_{v}(p)$ is a regular curve.

For this it is sufficient to show that $\bar{h}_{v}^{\prime}(p)(t) \neq 0$ for $t \geqq J_{0}$. For such $t$ we can write $\bar{h}_{v}^{\prime}(p)(t)=A_{1}+A_{2}$ where

$$
A_{1}=\bar{h}^{\prime}(p)(t)+s^{\prime} \beta^{\prime}(s)\left|h_{v}(p)-h_{0}(p)\right| u(p)
$$

and

$$
A_{2}=2 s^{\prime} s\left[h_{v}(p)-h_{0}(p)\right] .
$$

We will divide the proof into two parts.

CASE I. $s \leqq y_{0}$ : We claim $\left|A_{1}\right| \geqq(9 / 10)\left|\bar{h}^{\prime}(p)(1)\right|$.

For a certain number $\Delta$,

$$
A_{1}=\bar{h}^{\prime}(p)(1)+\Delta u(p)-\left(\bar{h}^{\prime}(p)(1)-\bar{h}^{\prime}(p)(t)\right)
$$

and then by the triangle inequality

$$
\left|A_{1}\right| \geqq\left|\bar{h}^{\prime}(p)(1)+\Delta u(p)\right|-\left|\bar{h}^{\prime}(p)(1)-\bar{h}^{\prime}(p)(t)\right| .
$$

By the choice of $J_{0}$ we obtain

$$
\left|A_{1}\right| \geqq\left|\bar{h}^{\prime}(p)(1)+u(p)\right|-\frac{1}{10}\left|\bar{h}^{\prime}(p)(1)\right| .
$$

Finally, since $u(p) \perp \bar{h}^{\prime}(p)(1)$ we have $\left|A_{1}\right| \geqq(9 / 10)\left|\bar{h}^{\prime}(p)(1)\right|$ as claimed.

On the other hand, by the choice of $y_{0}$ (since $s \leqq y_{0}$ ) 


$$
\left|A_{2}\right| \leqq s^{\prime}(1 / 3)\left|\bar{h}^{\prime}(p)(1)\right|\left(1-J_{0}\right)=(1 / 3)\left|\bar{h}^{\prime}(p)(1)\right| .
$$

From the triangle inequality it follows that $\bar{h}_{v}^{\prime}(p)(t) \neq 0$.

CASE II. $s \geqq y_{0}$ : We use a lemma.

Lemma 4.3. Let $a, b$, and $c$ be vectors in $E^{n}$ such that $|b|<(1 / 10)|a|$ and $c \perp a$. Let $v$ be a scalar, $v>4$. Then the inequality $|a+b+v c|>|3 c|$ holds.

Proof. Since

$$
|a+b+v c| \geqq|a+v c|-|b|
$$

it is sufficient to show

$$
|a+v c|^{2} \geqq(|3 c|+|b|)^{2}
$$

or using the fact that $c \perp a$

$$
|a|^{2}+|v c|^{2} \geqq 9|c|^{2}+6|b||c|+|b|^{2} .
$$

Since $v \geqq 4$ it is sufficient to show

$$
|a|^{2} \geqq-7|c|^{2}+\left.6|b| c|+| b\right|^{2} .
$$

This is easily checked considering separately the two cases

$$
|c| \leqq|b| \text { and }|c|>|b| \text {. }
$$

It follows from 4.3 that

$$
\left|A_{1}\right| \geqq 3 s^{\prime}\left|h_{v}(p)-h_{0}(p)\right| \text { taking } \bar{h}^{\prime}(p)(1)=a,
$$

$\bar{h}^{\prime}(p)(1)-\bar{h}^{\prime}(p)(t)=b, s^{\prime}\left|h_{v}(p)-h_{0}(p)\right| u=c$, and $\beta^{\prime}(s)=v$. By the choice of $J_{0},|b|<1 / 10|a|$ and since $s \geqq y_{0}, v \geqq 4$.

On the other hand $\left|A_{2}\right| \leqq 2 s^{\prime}\left|h_{v}(p)-h_{0}(p)\right|$. Then by the triangle inequality $\bar{h}_{v}^{\prime}(p)(t) \neq 0$. This finishes the proof of $(6)$.

Properties (1), (4), (5) and (6) imply that $\bar{h}_{v}(p)$ is really an element of $E$, (2) says that $\bar{h}_{v}$ covers $h_{v}$ and (3) that $\bar{h}_{v}$ is a homotopy of $\bar{h}$. Therefore we have proved 3.1.

5. Proof of Proposition 3.2. Let $U_{0}$ be a coordinate neighborhood on $M$, and let $V=T\left(U_{0}\right)$. By the argument used to prove Proposition 1.3, it is sufficient to prove 3.2 for the case where $\left\{f_{v}(p) \mid v \in I, p \in P\right\} \subset V$. The notation and conventions of the last section will be continued.

Let $\pi_{2}: T_{0}\left(U_{0}\right)=E^{n} \times E^{n} \rightarrow E^{n}$ be the projection onto the second factor. Then $\pi_{2}\left(T\left(U_{0}\right)\right) \subset S^{n-1}$. The angle between two vectors of $S^{n-1}$ is a continuous function of the vectors. This fact, together with the compactness of $P$, justifies the following choice. Pick $\epsilon>0$ such that for all $p \in P$ and $\left|v-v^{\prime}\right| \leq \epsilon$, the angle (measured in radians) between $\pi_{2} f_{v}(p)$ and $\pi_{2} f_{v^{\prime}}(p)$ is less than $1 / 10$.

For $v \leq \epsilon$ let $\alpha_{v}(p)$ be the oriented angle from $\pi_{2} f_{0}(p)$ to $\pi_{2} f_{v}(p)$. By our choice of $\epsilon, \alpha_{v}(p)<1 / 10$.

For $v \leqq \epsilon$, and $t \in I$, we will define $Q_{v}(p, t)$ to be the following rotation of 
$E^{n}$; that is, we are defining a map $Q_{v}: P \times I \rightarrow R_{n}$ where $R_{n}$ is the rotation group of $E^{n}$. If $\pi_{2} f_{0}(p)=\pi_{2} f_{v}(p)$ let $Q_{v}(p, t)=e$, the identity rotation. Otherwise let $Q_{v}(p, t)$ rotate $V$, the unique plane determined by $\pi_{2} f_{0}(p)$ and $\pi_{2} f_{v}(p)$, through the angle $t \alpha_{v}(p)$ and leave $V \perp$, the orthogonal complement of $V$, fixed.

That $Q_{v}(p, t)$ is continuous in $v, p$, and $t$ and has a continuous first derivative in $t, Q_{v^{\prime}}(p, t)$, is easily seen. Later, in fact, we will have occasion to compute this derivative.

Choose $J, 0 \leqq J<1$, such that

$$
\{\bar{f}(p)(t) \mid p \in P, t \in[J, 1]\} \subset U_{0} .
$$

Lemma 5.1. There exists a $J_{0}$ with $J \leqq J_{0}<1$ such that for all $p \in P$ and $t \in\left[J_{0}, 1\right]$,

$$
\left|\frac{\bar{f}(p)(t)-\bar{f}(p)(1)}{J_{0}-1}\right| \leqq \frac{4}{3}\left|\bar{f}^{\prime}(p)(1)\right| .
$$

Proof. It follows from the definition that

$$
\bar{f}^{\prime}(p)(1)=\lim _{t \rightarrow 1} \frac{\bar{f}(p)(t)-\bar{f}(t)(1)}{t-1}
$$

so by the compactness of $P$ there exists a $J_{0}$ with $J \leqq J_{0}<1$ such that for $t \in\left[J_{0}, 1\right]$,

$$
\left|\frac{\bar{f}(p)(t)-\bar{f}(p)(1)}{t-1}-\bar{f}^{\prime}(p)(1)\right| \leqq \frac{1}{3}\left|\bar{f}^{\prime}(p)(1)\right| .
$$

Then by the triangle inequality

$$
\left|\frac{\bar{f}(p)(t)-\bar{f}(p)(1)}{t-1}\right| \leqq \frac{4}{3}\left|\bar{f}^{\prime}(p)(1)\right| .
$$

Also clearly for $J_{0} \leqq t \leqq 1$,

$$
\left|\frac{\bar{f}(p)(t)-\bar{f}(p)(1)}{J_{0}-1}\right| \leqq\left|\frac{\bar{f}(p)(t)-\bar{f}(p)(1)}{t-1}\right| .
$$

These last two inequalities yield the lemma.

Choose $J_{0}$ by 5.1 and such that also

$$
\left|\bar{f}^{\prime}(p)(1)-\bar{f}^{\prime}(p)(t)\right|<(1 / 10) \min \left\{\left|f^{\prime}(p)(1)\right| \mid p \in P\right\}
$$

holds for all $p \in P$ and $t \in\left[J_{0}, 1\right]$. Then for $v \leqq \epsilon, \bar{f}_{v}$ is defined as follows.

For $0 \leqq t \leqq J_{0}$ set $\bar{f}_{v}(p)(t)=\bar{f}(p)(t)$.

For $J_{0} \leqq t \leqq 1$ let $s=s(t)=\left(t-J_{0}\right) /\left(1-J_{0}\right)$; then set

$$
\bar{f}_{v}(p)(t)=[\bar{f}(p)(t)-\bar{f}(p)(1)] Q_{v}(p, s)\left[e+\left(s^{2}-s\right) Q_{v}^{\prime}(p, 0)\right]+\bar{f}(p)(1) .
$$

Here $Q_{v}(p, s), Q_{v}^{\prime}(p, 0)$ and $e$ are to be considered as transformations acting 
on the right. The curve $\bar{f}(p)$ is taken distinguished, but in general, $\bar{f}_{v}(p)$ will not be.

The following properties of the covering homotopy can be quickly checked.

(1) $\bar{f}_{v}(p)(t)$ is continuous in $v, p$, and $t$.

(2) $\bar{f}_{0}(p)=\bar{f}(p)$.

(3) $\bar{f}_{v}(p)$ is stationary on a subpolyhedron $A$ of $P$ if $f_{v}(p)$ is.

The first derivative of $\bar{f}_{v}(p)$ can be computed as follows for $t \geqq J_{0}$ :

$$
\begin{aligned}
\bar{f}_{v}^{\prime}(p)(t)= & \bar{f}^{\prime}(p)(t) Q_{v}(p, s)\left[e+\left(s^{2}-s\right) Q_{v}^{\prime}(p, 0)\right] \\
+ & s^{\prime}[\bar{f}(p)(t)-\bar{f}(p)(1)]\left\{Q_{v}^{\prime}(p, s)\left[e+\left(s^{2}-s\right) Q_{v}^{\prime}(p, 0)\right]\right. \\
& \left.+Q_{v}(p, s)(2 s-1) Q_{v}^{\prime}(p, 0)\right\} .
\end{aligned}
$$

Using this it can be further checked that:

(4) $\bar{f}_{v}(p)(t)$ is differentiable.

(5) $\bar{f}_{v}^{\prime}(p)(0) /\left|\bar{f}_{v}^{\prime}(p)(0)\right|=x_{0}$ in the sense of $\S 2$.

(6) $\bar{f}_{v}^{\prime}(p)(1) /\left|\bar{f}_{v}^{\prime}(p)(1)\right|=f_{v}(p)$ again in the sense of $\S 2$.

The following requires proof:

(7) The curve $\bar{f}_{v}(p)(t)$ is regular.

For the proof of $(7)$ we will use:

Lemma 5.2. Let $0 \leqq t \leqq 1$. Then (a) the transformation $Q_{v}^{\prime}(p, t)$ reduces the magnitude of a vector to less than $1 / 10$ of its original magnitude and (b) the transformation $e+\left(t^{2}-t\right) Q_{v}^{\prime}(p, 0)$ does not change the magnitude of a vector by a factor of more than $1 / 10$.

Proof. For given $p$ and $v$ let coordinates $x_{1}, \cdots, x^{n}$ of $E^{n}$ be chosen so that $V$ (from the definition $\left.Q_{v}(p, t)\right)$ is the $x_{1}-x_{2}$ plane and the direction of $\pi_{2} f_{0}(p)$ coincides with the $x_{2}$ axis. Then with this system suitably oriented $Q_{v}(p, t)$ can be represented in the matrix form,

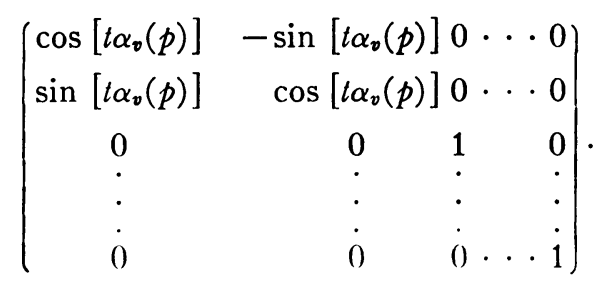

Then $Q_{v}^{\prime}(p, t)$ will be of the form,

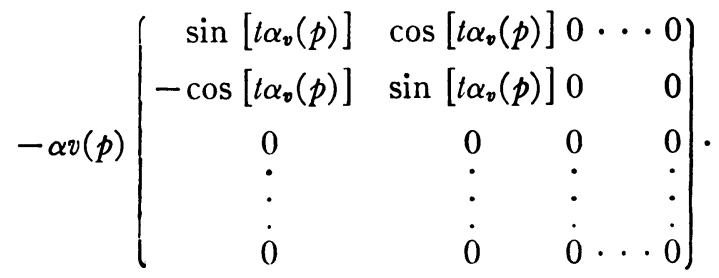


If $\beta_{1}, \beta_{2}, \cdots, \beta_{n}$ are the components of a vector $\beta$ in the above system then

$$
\left|\beta Q_{v}^{\prime}(p, t)\right|=\left|\alpha_{v}(p)\right|\left[\left(\beta_{1}\right)^{2}+\left(\beta_{2}\right)^{2}\right]^{1 / 2} \leqq\left|\alpha_{v}(p)\right||\beta| .
$$

This yields (a) since $\left|\alpha_{v}(p)\right|<1 / 10$. (b) follows from (a) and 5.2 is proved.

To prove (7) it is clearly sufficient to show that $\bar{f}_{v}^{\prime}(p)(t) \neq 0$ for $t \geqq J_{0}$.

Let $\bar{f}_{v}^{\prime}(p)(t)=A_{1}+A_{2}$ where

$$
A_{1}=\bar{f}^{\prime}(p)(t) Q_{v}(p, s)\left[e+\left(s^{2}-s\right) Q_{v}^{\prime}(p, p)\right]
$$

and

$$
\begin{aligned}
A_{2}=\left[\frac{\bar{f}(p)(t)-\bar{f}(p)(1)}{J_{0}-1}\right]\left\{Q_{v}^{\prime}(p, s)\left[e+\left(s^{2}-s\right) Q_{v}^{\prime}(p, 0)\right\rfloor\right. \\
\\
\left.+Q_{v}(p, s)(2 s-1) Q_{v}^{\prime}(p, 0)\right\} .
\end{aligned}
$$

From Lemma 5.2(b) and the fact that $Q_{v}(p, s)$ does not change the magnitude of a vector, it follows that $\left|A_{1}\right| \geqq(9 / 10)\left|\bar{f}^{\prime}(p)(t)\right|$. Then by the choice of $J_{0},\left|A_{1}\right| \geqq(8 / 10)\left|\bar{f}^{\prime}(p)(1)\right|$.

On the other hand, by Lemma 5.2 one easily obtains

$$
\left|A_{2}\right| \leqq 3 / 10\left|\frac{\bar{f}(p)(t)-\bar{f}(p)(1)}{J_{0}-1}\right| .
$$

Then from the choice of $J_{0}$ (see 5.1) it follows that $\left|A_{2}\right| \leqq(4 / 10)\left|\bar{f}^{\prime}(p)(1)\right|$. By the triangle inequality the inequalities on $\left|A_{1}\right|$ and $\left|A_{2}\right|$ yield $\bar{f}_{v}^{\prime}(p)(t) \neq 0$ and hence (7).

Properties (1), (4), (5) and (7) imply that $\bar{f}_{v}(p)$ really belongs to $E$, (2) says that $\bar{f}_{v}$ is a homotopy of $\bar{f}$ and (6) that $\bar{f}_{v}$ covers $f_{v}$. Lastly, (3) is the stationary property demanded by 3.2. Thus $\bar{f}_{v}$ is a satisfactory covering homotopy for $v \leqq \epsilon$.

The above construction may be repeated if $\epsilon<1$ using $\bar{f}_{\mathrm{e}}(p)$ instead of $\bar{f}(p)$ and using a new value for $J_{0}$, if necessary. This yields a covering homotopy for $v \leqq 2 \epsilon$. Iteration yields a full $\bar{f}_{v}$ and the proposition is proved.

6 . On the topology of the fiber $\Gamma$. We recall some definitions and theorems of [11]. Let $X$ be a space and $x_{0} \in X$. The path space of $X$ written $E_{x_{0}}(X)$ or sometimes $E_{x_{0}}$ is the space of all curves (or paths) on $X$ which start at $x_{0}$, with the compact open topology. Define $p: E_{x_{0}} \rightarrow X$ by sending a path onto its endpoint, i.e., let $p(f)=f(1)$. The loop space of $X$ at $x_{0}, p^{-1}\left(x_{0}\right)$ is denoted by $\Omega(X)$ or $\Omega$. It is shown in [11, pp. 479-481] that $\left(E_{x_{0}}, p, X\right)$ has the CHP and that $E_{x_{0}}$ is contractible.

Using the notation of the previous sections let $M$ be a manifold, $T=T(M)$, $E=E(M)$ and $\Gamma=\Gamma(M)=\pi^{-1}\left(x_{0}\right)$. Define a map $\phi: E \rightarrow E_{x_{0}}(T)$ by $\phi(g)(t)$ $=g^{\prime}(t) /\left|g^{\prime}(t)\right|$ for $g \in E$. It can be seen that $\phi$ is continuous as follows. Let $E^{\prime}$ be the set $E_{x_{0}}(T)$ endowed with the metric topology

$$
d^{*}(f, g)=\max \{d[f(t), g(t)] \mid t \in I\} .
$$


Then $\phi$ can be factored through $E^{\prime}$ by maps $\phi_{1}: E \rightarrow E^{\prime}$ and $\phi_{2}: E^{\prime} \rightarrow E_{x_{0}}$, where $\phi_{2}$ is the identity. It is well known that the metric topology and the compact open topology are equivalent on $E_{x_{0}}$. See for example $[4$, p. 55]. Hence $\phi_{2}$ is continuous. From the topology of $E$ it follows that $\phi_{1}$ is continuous. Thus $\phi$ is continuous. Let $\phi$ be $\phi$ restricted to $\Gamma$. Then $\bar{\phi}(\Gamma) \subset \Omega$. This map is the same as $\Phi$ of the Introduction.

A map between two spaces is a weak homotopy equivalence [after 10, p. 299 ] if it induces an isomorphism of the homotopy groups of the spaces. The following theorem is well-known. A proof can be found in [14, p. 113].

THEOREM 6.1. A weak homotopy equivalence induces isomorphisms of the singular homology groups of the spaces involved.

Theorem $C$ and 6.1 yield that $\bar{\phi}$ induces isomorphisms of the singular homology groups of $\Gamma$ and $\Omega$. This is of interest because a certain amount of attention has been given to the problem of determining the singular homology of loop spaces. For example see [11] and [15].

The proof of Theorem $\mathrm{C}$ requires the following lemma.

Lemma 6.2. If $M$ is a manifold the space $E(M)$ is homotopically trivial.

Proof. Consider first the case where $M$ is Euclidean $n$-space $E^{n}$. Assume $\pi_{1} x_{0}$ to be the origin of a coordinate system of $E^{n}$ and let $\pi_{2} x_{0}=\bar{x}_{0}$ where $\pi_{2}$ is the projection of $T=E^{n} \times S^{n-1}$ onto $S^{n-1}$.

For some $k \geqq 0$ let $f: S^{k} \rightarrow E$ be given. To prove the lemma for $E^{n}$ it is sufficient to show that $f$ is homotopic to a constant. Since $S^{k}$ is compact we can choose $J>0$ close enough to 0 so that for all $p \in S^{k}$ and $t \in[0, J]$,

$$
\left|f^{\prime}(p)(t)-f^{\prime}(p)(0)\right|<\left|f^{\prime}(p)(0)\right| \text {. }
$$

Then for $v \in[0,1 / 2]$ let $f_{v}(p)(t)=f(p)(t-2(1-J) v t)$. The curve $f(p)$ is to be distinguished, but $f_{v}(p)$ will not be, in general. This homotopy merely contracts $f(p)$ into a curve whose tangent is fairly close to a constant.

Define $e(t)$ as the fixed path of $E$ given by $\bar{x}_{0} t$. Then for $v \in[1 / 2,1]$ define

$$
f_{v}(p)(t)=(2-2 v) f_{1 / 2}(p)(t)+(2 v-1) e(t) .
$$

where $f_{1 / 2}(p)(t)$ is the nondistinguished curve given by the previous homotopy.

It can be checked that $f_{v}(p)$ is really contained in $E$, that $f_{0}(p)=f(p)$ and that $f_{1}(p)=e$. It is the selection of $J$ that yields the necessary regularity of $f_{r}(p)$ for $v \geqq 1 / 2$.

We have proved the lemma for $M=E^{n}$. The proof for a general $M$ goes as follows. As before, let $f: S^{k} \rightarrow E$. Now for $v \leqq 1 / 2$ let $f_{v}(p)$ be a "shortening" of $f(p)$ so that for all $p \in S^{k}, f_{1 / 2}(p)$ lies in a certain coordinate neighborhood about $x_{0}$. For $v \in[1 / 2,1]$ the homotopy is the same as the total homotopy for $E^{n}$. q.e.d.

Theorem $\mathrm{C}$ is proved as follows. 
From the definition of $\phi$ it follows easily that $\phi$ commutes with the identity of $T$, i.e., $p \phi=\pi$ or

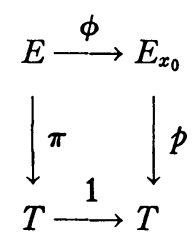

commutes. Then, $\phi$ induces a homomorphism of the homotopy sequence of $E$ into that of $E_{x_{0}}$. We have the following commutative diagram with the horizontal sequences exact.

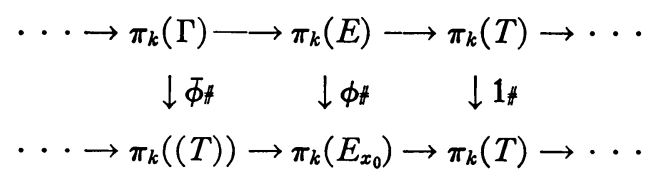

From $\pi_{k}(E)=\pi_{k}\left(E_{x_{0}}\right)=0$ for all $k$ (using 6.2), it follows that $\phi_{\#}$ is an isomorphism for all $k$. This proves Theorem C.

7. Classes of regular curves on a manifold. Two regular curves on a manifold $M$ are said to be regularly homotopic if they are homotopic and the homotopy $g_{v}: I \rightarrow M$ can be chosen such that for each $v \in I, g_{v}$ is a regular curve, $g_{v}^{\prime}(0)=g_{0}^{\prime}(0), g_{v}^{\prime}(1)=g_{0}^{\prime}(1)$, and $g_{v}^{\prime}(t)$ depends continuously on $v$. A regular curve $g$ on $M$ will be called closed if $g^{\prime}(0)=g^{\prime}(1)$. It will be said to be at a point $y_{0}$ of $T$ if $g^{\prime}(0) /\left|g^{\prime}(0)\right|=y_{0}$. Two closed regular curves on $M$ are freely regularly homotopic if they are homotopic and the homotopy $g_{v}: I \rightarrow M$ can be chosen so that for each $v \in I, g_{v}$ is a regular closed curve. Regular homotopy (free regular homotopy) is an equivalence relation and a class (free class) of regular curves on $M$ will mean an equivalence class with respect to this relation.

M. Morse has investigated the behavior of locally simple sensed closed curves (or $L$ - $S$-curves) under $L$-S-deformations. For definitions and discussion see $[7 ; 8 ; 9]$. In these articles he classified $L$-S-curves on closed 2 -manifolds and $E^{2}$ in to equivalence classes under $L-S$ deformations. He has noted the similarity between this study and the classification of closed regular curves with free regular homotopies playing the role of $L-S$-deformations. The results of this section are parallel to Morse's.

From the definition of regular homotopy it follows that if $M$ is a manifold two curves of $\Gamma(M)$ are regularly homotopic if and only if they lie in the same arcwise connected component of $\Gamma$, i.e., in the same element of $\pi_{0}(\Gamma)$. Using this fact Theorem $\mathrm{A}$ is the case $n=0$ of the following:

THEOREM 7.1. If $M$ is a manifold, there exists an isomorphism $q$ from $\pi_{n}(\Gamma(M))$ to $\pi_{n+1}(T(M))$. 
Proof. Let $\bar{\phi}_{\sharp}: \pi_{n}(\Gamma(M)) \rightarrow \pi_{n}(\Omega(T))$ be the isomorphism of Theorem C, and $s: \pi_{n}(\Omega(T)) \rightarrow \pi_{n+1}(T)$ be the Hurewicz isomorphism [16, p. 210]. The

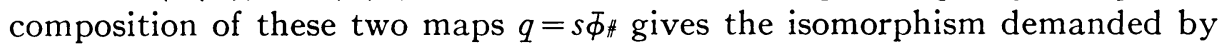
the theorem. The rest of this section will be devoted to special cases of Theorem $\mathrm{A}$.

THEOREM 7.2. Let the dimension of a manifold $M$ be greater than 2 and $x_{0} \in T(M)$. Then two regular closed curves on $M$ at $x_{0}$ are regularly homotopic if and only if they are homotopic with fixed end points.

As we shall see, this theorem is far from true for 2-manifolds.

Proof of 7.2. The "only if" part is immediate from the definition of regular homotopy.

Consider the exact homotopy sequence of $T$. Then

$$
\pi_{1}\left(S^{n-1}\right) \rightarrow \pi_{1}(T) \stackrel{\pi_{1 \sharp}}{\longrightarrow} \pi_{1}(M) \rightarrow \pi_{0}\left(S^{n-1}\right)
$$

is exact. Since $\pi_{1}\left(S^{n-1}\right)=\pi_{0}\left(S^{n-1}\right)=0$ for $n>2, \pi_{1 \sharp}: \pi_{1}(T) \approx \pi_{1}(M)$. With this, Theorem A yields that $\pi_{1 *} q: \pi_{0}(\Gamma) \approx \pi_{1}(M)$. Moreover, from the definitions of $\pi_{1}$ and $q$ we can consider $\pi_{1: H} q$ to be just the map induced by sending a curve into itself. Then 7.2 follows immediately from the definitions of $\pi_{0}(\Gamma)$ and $\pi_{1}(M)$.

Regular curve classes on some 2-manifolds will now be investigated.

(a) The plane. The following definition is due to Whitney [17]: If $f$ is a regular closed curve in $E^{2}$, its rotation number $\gamma(f)$ is the total angle which $\pi_{2} f^{\prime}(t)$ turns as $t$ traverses $I$. The function

$$
f^{*}(t)=\pi_{2} f^{\prime}(t) /\left|\pi_{2} f^{\prime}(t)\right|
$$

is a map of $I$ into the unit circle. $\gamma(f)$ is $2 \pi$ times the degree of this map.

TheOREM 7.3 (Whitney-Graustein). Two regular closed curves on the plane are freely regularly homotopic if and only if they have the same rotation number.

Proof. Because a translation of a regular closed curve in the plane is a free regular homotopy and preserves its rotation number it is sufficient to consider curves of $\Gamma\left(E^{2}\right)$.

Consider the isomorphisms

$$
\pi_{0}(\Gamma) \stackrel{q}{\rightarrow} \pi_{1}(T) \stackrel{\pi_{2 \sharp}}{\rightarrow} \pi_{1}\left(S^{1}\right)
$$

where $\pi_{2}$ is the projection of $T=E^{2} \times S^{1}$ onto $S^{1}$. Let $f$ be the element of $\Gamma$ represented by $e^{2 \pi i t}$ in complex coordinates such that the base point $x_{0}$ of $\Gamma$ is the vector $2 \pi i$ whose base point is the complex number 1 . If $h \in \Gamma, \bar{h}$ will be the element of $\pi_{0}(\Gamma)$ containing $h$. Then $\pi_{2 \sharp} q(\bar{f})$ will be a generator of 
$\pi_{1}\left(S^{1}\right)$ say $e$. If $g$ is any element of $\Gamma, \pi_{2 f} q(\bar{g})$ will be of the form $m e$. From the definition of rotation number it follows that $m$ is the rotation number of $g$. Since $\pi_{2 f} q$ is an isomorphism onto, this proves 7.3.

For the case of $L$-S-curves in the plane see [7].

(b) The 2-sphere $S^{2}$. It is known that $\pi_{1}\left(T\left(S^{2}\right)\right)$ is cyclic of order 2; for example see [13]. Hence, by Theorem A we can put regular closed curves of $S^{2}$ at a point $x_{0}$ into two classes under regular homotopy equivalence. For the case of $L$-S-curves on $S^{2}$, see [8].

(c) The torus $T^{2}$. From the exact homotopy sequence of the tangent bundle $T\left(T^{2}\right)$, it can be deduced that $\pi_{1}\left(T\left(T^{2}\right)\right.$ ) is $Z+Z+Z$ ( $Z$ is the infinite cyclic group). Then similar remarks to those of (b) apply.

(d) The projective plane $P^{2}$. It can be proved that $\pi_{1}\left(T\left(P^{2}\right)\right)$ is cyclc of order four. Hence, there are four classes of regular closed curves at a point $x_{0}$ on $P^{2}$. For the case of $L$-S-curves see [9].

REMARK. By taking $\Gamma$ as a fiber over a different point of $T$ one can obtain results similar to those of Sections 6 and 7 for nonclosed curves.

8. Regular curves perpendicular to a submanifold. Let $N$ be a regularly imbedded submanifold of a manifold $M$ and let $T$ be the unit tangent manifold of $M$. Let $V$ be the normal bundle of $N$ with respect to $M$; that is, $V$ is the subspace of $T$ which consists of all vectors which have their base points in $N$ and are normal to $N$. Let $x_{0}$ be a point of $V$ and $\Omega$ be the loop space of $T$ at $x_{0}$. Denote by $\Omega_{V}$ the subspace of $E_{x_{0}}(T)$ which consists of the paths ending in $V$.

Let $\Gamma_{N}$ be the subspace of $E(M)$ of curves whose final tangent is in $V$; i.e., $\Gamma_{N}=\pi^{-1}(V)$ where $\pi$ is the map of Theorem B. Let $\pi$ restricted to $\Gamma_{N}$ be still denoted by $\pi$. Then we have:

Theorem 8.1. The triple $\left(\Gamma_{N}, \pi, V\right)$ has the CHP.

Proof. This theorem is an easy consequence of Theorem B. In fact, let the homotopy $h_{v}: P \rightarrow V$ be given with $\bar{h}: P \rightarrow \Gamma_{N}$ covering $h_{0}$. Theorem B yields a covering homotopy $\bar{h}_{v}: P \rightarrow E$. But since $\bar{h}_{v}$ covers $h_{v}$ we have that $\bar{h}_{v}(p) \in \Gamma_{N}$ for all $v \in I$ and $p \in P$ and so $\bar{h}_{v}: P \rightarrow \Gamma_{N}$. This proves 8.1.

Similarly, $\left(\Omega_{V}, p, V\right)$ has the CHP. Let $\tilde{\phi}$ be the map $\phi$ of $\S 6$ restricted to $\Gamma_{N}$. Then

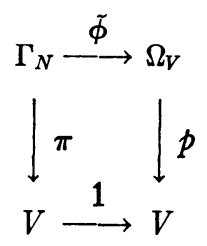

commutes so $\tilde{\phi}$ induces a homomorphism of the exact sequence of $\Gamma_{N}$ into that of $\Omega_{V}$. We have 


$$
\begin{aligned}
& \cdots \rightarrow \pi_{k}(\Gamma) \rightarrow \pi_{k}\left(\Gamma_{N}\right) \rightarrow \pi_{k}(V) \rightarrow \cdots \\
& \downarrow \tilde{\phi}_{\#} \quad \downarrow \tilde{\phi}_{\#} \quad \downarrow 1 \# \\
& \cdots \rightarrow \pi_{k}(\Omega) \rightarrow \pi_{k}\left(\Omega_{V}\right) \rightarrow \pi_{k}(V) \rightarrow \cdots
\end{aligned}
$$

By Theorem C, $\bar{\phi}_{\sharp}$ is an isomorphism onto and, of course, $1_{\sharp}$ is the identity isomorphism. From this and the "Five" Lemma [2, p. 16], it follows that $\tilde{\phi}_{\sharp}$ is also an isomorphism onto. We have proved:

TheOREM 8.2. The map $\tilde{\phi}: \Gamma_{N} \rightarrow \Omega_{V}$ defined above is a weak homotopy equivalence. Hence, by Theorem 6.1 it induces an isomorphism between the singular homology groups of $\Gamma_{N}$ and $\Omega_{V}$.

9. Integral curves of a 1-form. Let $T(M)$ be the unit tangent bundle of a 2-manifold $M$ and $\pi: T(M) \rightarrow M$ the projection. If $d \pi$ is the differential of $\pi$ and $v \in M_{m}$, the tangent space of $M$ at $m$, then $d \pi_{(0, m)}^{-1}(v)$ spans a two dimensional subspace containing the vertical. Hence there exists a 1 -form $\omega_{0} \neq 0$ on $T(M)$ annihilating this distribution of planes. If $f$ is a regular curve on $M$ then $\phi(f)$ (see the Introduction and Section 6) is an integral curve of $\omega_{0}$. One might hope to get a characterization of regular curves this way. Unfortunately, however, $\omega_{0}$ admits integral curves which are not the images under $\phi$ of regular curves. A curve lying in a single fiber of $T(M)$ is such an example. Thus it is not sufficient for the study of regular curves on a 2-manifold $M$ to study integral curves of $\omega_{0}$ on $T(M)$. However, there is still the question as to what can be said about integral curves of $\omega_{0}$. This section was written as an attempt to answer this question.

Throughout the rest of $\S 9$ we will assume that $M$ is a given manifold of dimension three. It seems very likely that the theory here generalizes to manifolds of higher dimension. However, because the treatment of 3-manifolds is so much simpler, we confine ourselves to this case.

A kind of curve essentially the same as the "stuckweise glatt" curves of [12] is considered here. A curve $f$ on $M$ is called a parametrized piecewise regular curve if there exist real numbers $t_{i}$ for $i=0,1, \cdots, k$ with $t_{0}=0, t_{k}=1$, and $t_{i}<t_{i+1}$ such that for each $i<k, f$ restricted to $\left[t_{i}, t_{i+1}\right]$ is either constant or regular in the sense that $\left|f^{\prime}(t)\right| \neq 0$ for $t \in\left[t_{i}, t_{i+1}\right]$. We say that such a curve is distinguished if its parameter is proportional to arc-length [12]. By changing the parameter one can associate to each parametrized piecewise regular curve a unique distinguished parametrized piecewise regular curve [see 12]. Two parametrized piecewise regular curves will be called equivalent if their associated distinguished curves are the same. A piecewise regular curve is an equivalence class of parametrized piecewise regular curves. Each such curve will have a unique distinguished representative. Oftentimes we will identify a piecewise regular curve with its distinguished representative.

As will be shown by an example at the end of the section, the theorems here hold only if some restriction is placed on the 1 -forms. 
Let $q_{0}$ be a fixed point of $M$, and let $\omega$ be a 1 -form on $M$ such that $\omega \wedge d \omega \neq 0$ on $M$. Denote by $E_{\omega}$ the space of all piecewise regular curves on $M$ which start at $q_{0}$ and are integral curves of $\omega$. Let $E_{\omega}$ be a metric space under the metric

$$
d(f, g)=\max \{\bar{d}(f(t), g(t)) \mid t \in I\}
$$

where $\bar{d}$ is any fixed metric on $M$. Define a map $p: E_{\omega} \rightarrow M$ by $p(g)=g(1)$. Let $p^{-1}\left(q_{0}\right)$ be denoted by $\Omega_{\omega}$.

\section{Theorem 9.1. The triple $\left(E_{\omega}, p, M\right)$ has the CHP.}

Roughly speaking, Theorem 9.1 is proved as follows. First, by a classical theorem, there are local coordinates $(x, y, z)$ about a point of $M$ such that in them $\omega$ assumes an especially simple form. Here the fact that $\omega \wedge d_{\omega} \neq 0$ is used. By Proposition 1.3 we reduce the proof, in a sense, to this local situation. Then the local coordinates thus obtained are used to write down explicitly the desired covering homotopy equations.

We break the definition of the covering homotopy curves into four parts according to values of the parameter $t$. In general, this curve will turn out to have a corner at $t=1 / 4, t=1 / 2$, and $t=3 / 4$. The first part of the constructed curve is merely a reparametrization of the given covering curve. Then the construction is such that at $t=1 / 2$, the $z$ coordinate of the covering homotopy has moved to a position over the $z$-coordinate of the given homotopy. At $t=3 / 4$ the $x$-coordinate has undergone a similar motion, and finally, at $t=1$, the $y$-coordinate of the covering homotopy projects into the $y$-coordinate of the given homotopy in $M$.

Proof of 9.1. Let $q \in M$. Take a coordinate neighborhood $U$ of $q$ with coordinates $(x, y, z)$. In $U$ we can write $\omega=P d x+Q d y+R d z$ where $P, Q$, and $R$ are differentiable functions of $x, y$ and $z$. Then

$$
\omega \wedge d \omega=\left(P P^{\prime}+Q Q^{\prime}+R R^{\prime}\right) d x \wedge d y \wedge d z
$$

in $U$ where

$$
P^{\prime}=R_{y}-Q_{z}, \quad Q^{\prime}=P_{z}-R_{x}, \quad R^{\prime}=Q_{x}-P_{y} .
$$

Hence $P P^{\prime}+Q Q^{\prime}+R R^{\prime} \neq 0$ in $U$ since $\omega \wedge d \omega \neq 0$. Then by a classical result of the theory of differential equations (see for example [3, p. 58]) there exist differentiable functions $u, v$ and $w$ of $x, y$, and $z$ defined in a neighborhood of $q$ such that $\omega=d u+v d w$. Furthermore $0 \neq \omega \wedge d \omega=d u \wedge d v \wedge d w$ so that $u, v$ and $w$ form a coordinate system in a neighborhood say $V$ of $q$. By 1.3 it is sufficient to prove the CHP for the triple $\left(p^{-1}(V), p, V\right)$. For convenience we will change the coordinates $u, v$, and $w$ into $x, y$, and $z$ respectively. So now $x$, $y$, and $z$ are coordinates of $V$ such that $\omega=d x+y d z$.

Let $h_{v}: P \rightarrow V$ be a given homotopy with $\bar{h}: P \rightarrow p^{-1}(V)$ covering $h_{0}$. We will construct a covering homotopy $\bar{h}_{v}: P \rightarrow p^{-1}(V)$. To describe these maps 
in the coordinate system $(x, y, z)$ we use the following notation:

$$
\begin{aligned}
h_{v}(p) & =\left(x_{v}(p), y_{v}(p), z_{v}(p)\right), \\
\bar{h}_{v}(p)(t) & =\left(\bar{x}_{v}(p)(t), \bar{y}_{v}(p)(t), \bar{z}_{v}(p)(t)\right) .
\end{aligned}
$$

Then : for $0 \leqq t \leqq 1 / 4$ let

$$
\bar{h}_{v}(p)(t)=\bar{h}(p)(4 t)
$$

for $1 / 4 \leqq t \leqq 1 / 2$ let $s=s(t)=4 t-1$ and let

$$
\begin{aligned}
& \bar{x}_{v}(p)(t)=x_{0}(p)-\left[z_{v}(p)-z_{0}(p)\right] y_{0}(p) s, \\
& \bar{y}_{v}(p)(t)=y_{0}(p), \\
& \bar{z}_{v}(p)(t)=z_{0}(p)+\left[z_{v}(p)-z_{0}(p)\right] s
\end{aligned}
$$

for $1 / 2 \leqq t \leqq 3 / 4$ let $s=s(t)=4 t-2$. Then let

$$
\begin{aligned}
\tilde{x}_{v}(p)= & x_{0}(p)-y_{0}(p)\left[z_{v}(p)-z_{0}(p)\right\rfloor, \\
\bar{x}_{v}(p)(t)= & \left(36 s^{5}-45 s^{4}-20 s^{3}+30 s^{2}\right)\left\lfloor x_{v}(p)-\tilde{x}_{v}(p)\right\rfloor \\
& +60\left|x_{v}(p)-\tilde{x}_{v}(p)\right|^{1 / 2} y_{0}(p)\left(s^{3}-s\right)+\bar{x}_{v}(p), \\
\bar{y}_{v}(p)(t)= & \operatorname{Sg}\left|x_{v}(p)-\bar{x}_{v}(p)\right|^{1 / 2}\left(s^{2}-s\right)+y_{0}(p), \\
& \operatorname{Sg}=\operatorname{sign} \text { of } x_{v}(p)-\tilde{x}_{v}(p), \\
\bar{z}_{v}(p)(t)= & -60\left|x_{v}(p)-\tilde{x}_{v}(p)\right|^{1 / 2}\left(s^{3}-s\right)+z_{v}(p)
\end{aligned}
$$

for $3 / 4 \leqq t \leqq 1$ let $s=s(t)=4 t-3$ and

$$
\begin{aligned}
& \bar{x}_{v}(p)(t)=x_{v}(p), \\
& \bar{y}_{v}(p)(t)=\left[y_{v}(p)-y_{0}(p)\right] s+y_{0}(p), \\
& \bar{z}_{v}(p)(t)=z_{v}(p) .
\end{aligned}
$$

In order to be sure that these equations define a satisfactory covering homotopy it must be checked that $(1) \bar{h}_{v}(p)$ is a curve in $E_{\omega}$ (for each $p$ and $v),(2) \bar{h}_{v}(p)$ is a homotopy of $\bar{h}(p)$ or $\bar{h}_{0}(p)=\bar{h}(p)$, and (3) $\bar{h}_{V}(p)$ covers $h_{V}(p)$ or $\bar{h}_{v}(p)(1)=h_{v}(p)$.

We will check $(1)$ first. It is easy to note that $\bar{h}_{v}(p)(0)=q_{0}$. Also, $\bar{h}_{v}(p)(t)$ is clearly continuous and piecewise regular between the values $t=0,1 / 4,1 / 2$, $3 / 4$, and 1 . It is necessary to check that $\bar{h}_{v}(p)(t)$ is well-defined at $t=1 / 4,1 / 2$, and $3 / 4$ since at each of these values $\bar{h}_{v}(p)(t)$ is defined in two different ways. By substituting these values of $t$ into the appropriate equations it can be seen that where the definitions overlap they agree. To complete the proof of (1) it needs to be shown that $\bar{h}_{v}(p)(t)$ satisfies $\omega=0$ or, in other words,

$$
\frac{d \bar{x}_{v}(p)(t)}{d t}+\bar{y}_{v}(p)(t) \frac{d \bar{z}_{v}(p)(t)=0}{d t}=0
$$


identically for all $v, p$, and $t$. This is trivial for $t \leqq 1 / 4$. For $1 / 4 \leqq t \leqq 1$ to prove that this differential equation is satisfied it is sufficient to make three computations, one for $1 / 4 \leqq t \leqq 1 / 2$, one for $1 / 2 \leqq t \leqq 3 / 4$ and one for $3 / 4 \leqq t \leqq 1$. These are not difficult and will be left for the reader.

To show (2) we set $v=0$, getting

$$
\begin{array}{ll}
\bar{h}_{0}(p)(t)=\bar{h}(p)(4 t) & 0 \leqq t \leqq 1 / 4, \\
\bar{x}_{0}(p)(t)=x_{0}(p), & 1 / 4 \leqq t \leqq 1, \\
\bar{y}_{0}(p)(t)=y_{0}(p) & \\
\bar{z}_{0}(p)(t)=z_{0}(p) . &
\end{array}
$$

This is a parametrized piecewise regular curve whose associated distinguished curve is exactly the given curve $\bar{h}(p)(t)$.

It is trivially checked that (3) holds. q.e.d.

The space $E_{\omega}$ is contractible to a point. The deformation accomplishing this is $D: E_{\omega} \times I \rightarrow E_{\omega}$ defined by $D(f, v)=f(v t)$. Define a map $i: E_{\omega} \rightarrow E_{q \bullet}(M)$ (see $\S 6$ ) by letting $i(f)$ be the distinguished representative of $f$. Then $i$ is continuous by the argument used to show that $\phi$ was continuous in $\$ 6$. Let $i$ be $i$ restricted to $\Omega_{\omega}$ where $\Omega_{\omega}=p^{-1}\left(q_{0}\right) \subset E_{\omega}$. The argument used in $\S 6$ to show that $\bar{\phi}: \Gamma \rightarrow \Omega(T)$ was a weak homotopy equivalence may now be used to show that $i$ : $\Omega_{\omega} \rightarrow \Omega(M)$ is also a weak homotopy equivalence. This proves Theorem D.

The following example shows how Theorems 9.1 and $\mathrm{D}$ fail for a form $\omega_{0}$ which is completely integrable. Let $M=E^{3}$ and $\omega_{0}=x d x+y d y+z d z$ in a given Cartesian coordinate system $(x, y, z)$ of $E^{3}$. Take for $q_{0}$ any point at distance $d>0$ from the origin of $E^{3}$. Then any integral curve of $\omega_{0}$ starting at $q_{0}$ stays on the surface of the 2-sphere $x^{2}+y^{2}+z^{2}=d^{2}$. Clearly, the conclusions of Theorems 9.1 and $D$ fail in this case. Actually, Theorem 9.1 is false for any 1 -form which is completely integrable at a certain point $q_{0} \in E^{3}$. For then short curves at $q_{0}$ must lie on a surface of $E^{3}$ and the covering homotopy property cannot possibly hold.

\section{BIBLIOGRAPHY}

1. S. Chern, Differentiable manifolds, Notes at University of Chicago, 1955.

2. S. Eilenberg and N. Steenrod, Foundations of algebraic topology, Princeton, 1952.

3. A. R. Forsyth, A treatise on differential equations, London, 1951.

4. S. Hu, Homotopy theory, Notes at Tulane, 1950.

5. W. Hurewicz, On the concept of a fiber space, Proc. Nat. Acad. Sci. U.S.A. vol. 55 (1955) pp. 956-961.

6. I. M. James and J. H. C. Whitehead, Note on fiber spaces, Proc. London Math. Soc. vol. 4 (1954) pp. 129-137.

7. M. Morse, Topological methods in the theory of functions of complex variables, Princeton, 1947.

8. - L-S-homotopy classes of locally simple curces, Annales de la Societe Polonaise de Mathematique vol. 21 (1948) pp. 236-256. 
9. - L-S-homotopy classes on the topological image of a projective plane, Bull. Amer. Math. Soc. vol. 55 (1949) pp. 981-1003.

10. H. Samelson, Groups and spaces of loops, Comment. Math. Helv. vol. 29 (1954) pp. 278-287.

11. J.-P. Serre, Homologie singuliere des espaces fibres, Ann. of Math. vol. 54 (1951) pp. 425-505.

12. Seifert and Threlfall, Variationsrechnung in Grossen, New York, 1948.

13. N. Steenrod, The topology of fiber bundles, Princeton, 1951.

14. G. W. Whitehead, Homotopy theory, Notes at Massachusetts Institute of Technology, 1054.

15. - On the homology suspension, Ann. of Math. vol. 62 (1955) pp. 254-268.

16. - On the Freudenthal theorems, Ann. of Math. vol. 57 (1953) pp. 209-228.

17. H. Whitney, On regular closed curves in the plane, Compositio Math. vol. 4 (1937) pp. 276-284.

\section{University of Chicago,}

Chicago, Ill.

UNIVERSITY OF MichigAN

AnN Arbor, Mich. 